\title{
Response to "Letter to the Editor" by Balta et al. entitled "Other inflammatory markers ought to be kept in mind when assessing the mean platelet volume in clinical practice"
}

\author{
Sahin Ulu
}

Published online: 9 May 2013

(c) Springer-Verlag Berlin Heidelberg 2013

\section{Dear Editor,}

MPV will be much more beneficial when used with other inflammatory markers of course.

However, in this article, our goal was to make useful a laboratory data which is routinely measured and available costless. We suggested that clinicians should not ignore MPV in terms of thromboembolic events while evaluating idiopathic sudden hearing loss.

In addition, in the letter by Balta et al. it was indicated that, MPV alone, cannot provide information about the state of inflammation. There may be a misunderstanding on this issue.
Because in our study, MPV was evaluated as a thromboembolic marker but not as an inflammatory marker.

Best regards,

Sahin Ulu.

S. Ulu $(\bowtie)$

Department of Otolaryngology, Faculty of Medicine, Afyone

Kocatepe University, 03200 Afyonkarahisar, Turkey

e-mail: drsahinulu@yahoo.com 